\title{
Psicoterapia de grupo en una unidad de agudos
}

RESUMEN: Se describe el proceso de puesta en marcha y desarrollo de una terapia de grupo en una Unidad de Agudos.

PALABRAS CLAVE: Psicoterapia de grupo, unidad de agudos, hospitalización breve.
SUMMARY: The starting process and development of a group therapy in an acute-care unit is described.

KEY WORDS: Inpatient psychotherapy group, acute-care unit, brief hospitalization.

Presentamos aquí la experiencia grupal que se está llevando a cabo desde marzo de 2007 en la Unidad de Hospitalización Breve del Hospital Son Llàtzer de Palma de Mallorca. Esta iniciativa surgió como manera de dar una respuesta a algunas de las necesidades observadas en los pacientes ingresados, y pudo ser puesta en marcha gracias a la incorporación de una psicóloga clínica en la plantilla.

La inclusión de sesiones de grupo dentro de las Unidades de Agudos es frecuente y su importancia está siendo reconocida de manera creciente en la literatura: Brabender (1). Muchos autores han puesto de manifiesto la pertinencia y utilidad de estos grupos en estas Unidades, como Yalom (2); Janssen (3); Brabender y Fallon (4); Galletero y otros (5); Oldham y Russakoff (6); Kibel (7); Powell (8); Farkas-Cameron (9); Gauron (10); Agazarian (11); Clunie (12); Rice y Rutan (13).

Los grupos de psicoterapia en las Unidades de agudos cumplen un papel fundamental para reducir el impacto de los factores potencialmente estresantes asociados a la hospitalización y a la situación de crisis en la que ingresan los pacientes, constituyéndose como un factor protector y necesario para disminuir la ansiedad de los pacientes y disminuir las proyecciones paranoides que están en la base de muchas actuaciones violentas (14). Este autor destaca la tensión tan fuerte que soportan los pacientes ingresados en una Unidad de Agudos, consecuencia de estar en una situación excepcional en sus vidas como es la de afrontar un entorno a menudo desconocido, rodeados de otras personas en crisis, sin un rol específico y claro que pueda facilitar su adaptación y proporcione la sensación de protección implícita en los grupos sociales. La adaptación resulta complicada porque además la persona está sometida a expectativas por parte del equipo terapéutico y carece de las ayudas habituales de su entorno.

Los pacientes hospitalizados experimentan dos tipos de crisis (15): la que les ha llevado al ingreso y la crisis más general de ser hospitalizado. En este contexto pueden percibir el tratamiento como presiones para abandonar sus defensas y amenazas a su autocontrol, por lo que los pacientes pueden desconfiar del perso- 
nal y manifestar ambivalencia sobre la necesidad de estar ingresados y dudan de que la hospitalización les pueda ayudar.

Es destacable también que además de ser un entorno desconocido con gente desconocida, percibidos muchas veces como hostil, es un entorno cuyas estructuras físicas distan de ser cálidas y acogedoras, e incluso pueden ser muy agobiantes si no disponen de un espacio abierto.

Debido a estas particularidades, los profesionales que trabajan en estas unidades con intervenciones grupales han tenido que modificar las técnicas clásicas de psicoterapia de grupo $(16 ; 3 ; 17)$, si bien hay experiencias utilizando modelos de grupo cerrado, con una selección previa de pacientes, y cuya estabilidad de miembros permite el desarrollo de la cohesión y por tanto el uso de técnicas y objetivos de la psicoterapia de grupo tradicional (1).

En las siguientes páginas se abordarán los objetivos de los grupos de pacientes ingresados, cómo es el proceso de iniciar un grupo de este tipo, las tareas del terapeuta y los distintos profesionales que participan en el grupo relacionadas con cada uno de los objetivos propuestos y de acuerdo con las diferentes fases de desarrollo por las que atraviesa el grupo, ilustrándolo con ejemplos y señalando los factores terapéuticos que justifican la inclusión de estos grupos en estas Unidades. Finalmente, se incluye un apartado en el que se perfilan las dificultades por las que puede atravesar el terapeuta.

\section{Objetivos del grupo}

Las características de las Unidades de Hospitalización, la situación de crisis en la que se encuentra el paciente ingresado y el estrés de la hospitalización van a condicionar los objetivos de los grupos que se pongan en marcha.

Yalom (17) describe de la siguiente manera el escenario clínico del grupo con pacientes hospitalizados, que coincide con el nuestro: hay una considerable rotación de pacientes; muchos pacientes asisten a la reunión de grupo tan sólo una o dos veces; hay una gran heterogeneidad en la psicopatología; todos los pacientes se sienten sumamente incómodos; hay muchos pacientes desmotivados en el grupo; el terapeuta no tiene tiempo de preparar o seleccionar pacientes; el terapeuta no tiene control sobre la composición del grupo; hay poca estabilidad de terapeutas; los pacientes ven a su terapeuta en otros menesteres, en el ámbito hospitalario, a lo largo del día; la terapia de grupo es tan sólo una de las terapias en las que participa el paciente; hay frecuentemente poca sensación de cohesión en el grupo; no hay tiempo para un reconocimiento gradual de patrones interpersonales sutiles, o para «trabajar por», y no hay oportunidad para centrarse en la transferencia del aprendizaje a la situación familiar. 
ORIGINALES Y REVISIONES

Leszcz (18), además de señalar algunas de las características anteriores, apunta como aspecto diferencial de estos grupos el que se hallen abiertos a la observación y el escrutinio del medio, así como la interacción de los miembros del grupo fuera de las sesiones, no sólo como algo que ocurre necesariamente por el hecho de ser una unidad cerrada, sino como algo a fomentar. En estas circunstancias, Vinogradov y Yalom (19) consideran que el objetivo fundamental de este tipo de grupos es que los pacientes logren recuperar el funcionamiento previo a la crisis.

Enumeremos los objetivos planteados para el grupo en el Hospital Son Llàtzer. En el apartado de «tareas del terapeuta de grupo y del personal de apoyo» veremos algunas estrategias y técnicas para lograr tales objetivos: a) Ofrecer un espacio de contención y estructura. b) Ofrecer un espacio de acogida y acompañamiento durante el ingreso. c) Permitir la expresión personal de cada paciente a través de la palabra. d) Fomentar la parte sana y los recursos personales de cada paciente. e) Abordaje de dificultades en las relaciones entre pacientes y entre pacientes y personal. f) Facilitar el establecimiento y el desarrollo de relaciones nutricias y estructurantes entre los miembros del grupo. g) Ayudar a los pacientes a entender su situación actual. h) Abordar cuestiones más profundas relacionadas con la historia biográfica de los miembros, si se dan las condiciones para que surja ese material y pueda ser elaborado por el grupo.

Aunque el grupo no esté pensado para ello, resulta muy útil en la tarea de esclarecimiento diagnóstico. De la misma manera, el grupo es también un espacio privilegiado para valorar las relaciones entre los diferentes elementos del sistema que conforma la Unidad entera: relaciones entre las mismas o diferentes categorías profesionales, la coordinación, la estructura de funcionamiento y organizativa. El grupo de terapia refleja simbólicamente el proceso dinámico de la Unidad $(20 ; 13 ; 2 ; 16)$ y viceversa, la puesta en marcha del grupo puede influenciar a estos sistemas de rango superior (21). La existencia del grupo supone una oportunidad para tomar conciencia de estos factores y contribuir de alguna manera al cambio hacia un clima lo más terapéutico posible.

\section{Factores previos y encuadre}

En los apartados anteriores hemos visto que realizar sesiones de grupo cubre necesidades importantes de los pacientes ingresados en las Unidades de Hospitalización Breve, por lo que cuando el profesional se plantea mejorar la asistencia de los pacientes no es raro que piense en incluir en la oferta de tratamientos un tipo u otro de experiencia grupal.

Una vez se tienen claros los objetivos -el «para qué» que guiará todos los pasos siguientes- es importante que todos los profesionales de la planta conozcan 
y puedan participar en el proceso de ir perfilando las condiciones en las que se va a desarrollar el grupo. El grupo es un elemento nuevo que se introduce en un sistema formado, y romperá temporalmente el equilibrio existente, exigiendo un reajuste general. Es necesario que todos los profesionales consideren el grupo como parte del programa de la unidad, para minimizar la influencia de lo que se consideran «factores extrínsecos que pueden entorpecer el grupo» (19). Para ello es importante que la psicoterapia de grupo sea incluida en la cartera de servicios que el Hospital ofrece a sus clientes y que se establezcan los medios mediante los cuales se va a informar a los usuarios de su existencia y fines. Hay que asegurarse de que existe congruencia entre el rol institucional que el Hospital da a la psicoterapia de grupo y la visión asistencial de la persona que lo conduce. Por otra parte hay que designar a la persona que va a asumir la responsabilidad de garantizar la infraestructura para que la psicoterapia de grupo sea posible.

En relación con la integración del grupo en la Unidad, como una parte importante de la atención al paciente, es necesario plantearse las siguientes cuestiones: ¿Cuál será la forma de conectar las actividades clínicas propias de la psicoterapia de grupo con los objetivos que el equipo terapéutico diseña para resolver la crisis del paciente? ¿Cómo puede cada terapeuta individual o el profesional de referencia del paciente beneficiarse de la psicoterapia de grupo para que su paciente mejore más y más pronto? ¿Cómo informará el terapeuta de grupo al equipo terapéutico de los progresos de los pacientes o de las incidencias en la dinámica grupal? ¿Cómo va a tener en cuenta el profesional de referencia los vínculos que establece el paciente en el grupo a la hora de entender su evolución en la unidad o a la hora de prepararle para el alta? ¿Cómo se van a tener en cuenta estos vínculos a la hora de diseñar el seguimiento del paciente? ¿Se va a tener o no una postsesión, con qué fines y quiénes participarán?

Hay otras cuestiones prácticas que decidir antes de iniciar el grupo: El encuadre: cuándo, durante cuánto tiempo, con qué frecuencia, dónde. ¿Qué profesionales entrarán en las sesiones y quién va a conducir el grupo? ¿Cuál será la función de cada profesional en el grupo (enfermería, auxiliares, psiquiatras, psicólogos, residentes y alumnos)? ¿Qué espacios habrá para la coordinación entre los distintos profesionales de la Unidad? ¿Cuáles serán los criterios de inclusión/exclusión y quién los decide para un paciente determinado? ¿Será un grupo voluntario u obligatorio para los pacientes? ¿Tendrá el paciente algún tipo de preparación antes de entrar en el grupo?

El grupo que pusimos en marcha comenzó realizándose en un principio a diario (tres días de mañana y dos de tarde), para posteriormente pasar a anular los de la tarde. Kaplan y Sadock (22) argumentan a favor del mayor número de sesiones posibles, ya que cuanto menos frecuentes son las sesiones, mayor es la necesidad de que el terapeuta estructure el grupo y sea activo.

Los profesionales que habitualmente entran en las sesiones son la conductora del grupo (una psicóloga clínica) y personal de apoyo (un/a enfermero/a y un/a 
ORIGINALES Y REVISIONES

auxiliar). También acuden a algunas sesiones alumnos de enfermería y psicología y residentes de psiquiatría o de familia, así como los/las psiquiatras de la planta, que son observadores o en ocasiones observadores participantes y personal de apoyo. Para evitar desproporción entre número de pacientes y número de profesionales, solemos limitar a cuatro el número de profesionales por sesión.

El único momento fijado para hablar sobre el grupo entre los profesionales es al final de cada sesión. Son apenas unos diez-quince minutos que pretenden servir para ventilar emociones, comentar la dinámica grupal y también la evolución o valoración del estado psicopatológico de los pacientes. Existen otros espacios informales para estos propósitos, espacios que van aumentando a medida que el grupo va teniendo más presencia en la Unidad.

El grupo está abierto a todos los pacientes, aunque se excluye a pacientes agitados o pacientes cuyo estado psicopatológico interfiere totalmente la realización del grupo. Cualquier profesional puede proponer la exclusión de un paciente para una sesión, y se suele realizar momentos antes de iniciar el grupo. Es muy raro que se excluya del grupo a alguien que está interesado en participar en él.

El grupo es voluntario, y el número de pacientes que suelen asistir es entre diez y quince. En la literatura se encuentran experiencias en que unas veces se aconseja que los grupos de pacientes ingresados sean voluntarios (22) y en otras que sean obligatorios (19). No hay tiempo para preparar al paciente antes de entrar en el grupo, sin embargo, desde el marco que trabajamos de «sesión única» de Yalom, los primeros minutos del grupo ayudan a la integración de todos los pacientes presentes. Este marco de trabajo supone considerar la vida del grupo como de una sola sesión. En nuestra experiencia es la forma de encuadrarlo, que se ajusta más a la realidad de las unidades de crisis con su fuerte rotación de pacientes, a las expectativas de los pacientes y a las del terapeuta.

\section{Tareas del terapeuta de grupo y el personal de apoyo}

Puesto que los objetivos del grupo son necesariamente específicos de este tipo de Unidad y de pacientes ingresados, las técnicas estándar de la terapia de grupo requieren una modificación para este contexto clínico (19). A continuación vamos a ver por medio de qué técnicas se pueden lograr cubrir los objetivos mencionados.

\section{Ofrecer un espacio de contención y estructura}

La forma en que se puede lograr que ese espacio grupal constituya un espacio de contención y estructura es, en un nivel muy básico, garantizar adecuados 
límites espaciales y temporales: la sala siempre presenta la misma disposición de sillas y mesas, la sesión se realiza con la puerta cerrada y se tiene especial cuidado en que si se dan interrupciones por pacientes que llegan tarde o abren la puerta sólo para ver qué hay, sean lo más cortas y menos perturbadoras posible.

También contribuye a la contención la existencia de normas explícitas que preserven la integridad personal y grupal. En los primeros minutos de la sesión se verbaliza que la norma fundamental del grupo es la de no agresividad física ni verbal hacia uno mismo o a los otros dentro del grupo, y se invita a salir del grupo a quien no se sienta capaz de mantenerlo. Cómo se maneja esto depende mucho de la situación concreta de la que se trate, pero en cualquier caso su abordaje constituye una prioridad cuando se da dentro del grupo.

Las sesiones están estructuradas por medio de una secuencia fija de acciones que hacen que la sesión sea de alguna manera predecible para aquellos que acuden más de una vez: breve explicación por parte del terapeuta de grupo del encuadre, objetivos del grupo y norma, así como de la estructura de la sesión.

Para conseguir dotar a la sesión de capacidad estructurante es necesario que el terapeuta de grupo sea muy activo. Imaginemos un inicio de sesión en el cual nadie habla, se miran unos a otros, es un silencio realmente angustiante para muchos pacientes. El terapeuta puede en este momento ayudar a comenzar al grupo disolviendo esa tensión planteando alguna pregunta, señalando la dificultad de hablar cuando hay tanto silencio o validando la angustia que produce ese momento. Puede invitar a algunos miembros a hablar, por ejemplo, preguntar a un paciente que se va de alta si quiere contar su experiencia del ingreso.

Dentro del arsenal técnico del terapeuta está la posibilidad de proponer ejercicios o tareas estructuradas. Como hemos visto, hay que procurar que los ejercicios estructurados que se propongan no despojen a los pacientes de un papel activo dentro del grupo, es decir, es esencial estructurar al grupo de tal manera que fomente la autonomía de funcionamiento de cada uno de los miembros, y no les impida poner en marcha y beneficiarse de sus habilidades interpersonales (19).

\section{Ofrecer un espacio de acogida y acompañamiento}

En la medida en la que el grupo consigue ofrecer este espacio el sujeto va a tener un modelo para dar un lugar a sus síntomas en su mundo interno. El apoyo genuino mostrado a través de la comprensión, aceptación y reconocimiento al paciente supone muchas veces la herramienta más potente para disminuir la angustia asociada a la hospitalización y la crisis.

Una de las tareas del terapeuta es minimizar el rechazo que pueda provocar la actitud de algunos pacientes en el grupo (19), por ejemplo, ofreciendo una explica- 
ORIGINALES Y REVISIONES

ción alternativa a su conducta o mediante preguntas que ayuden al paciente a mostrar aspectos más positivos de su personalidad. No es raro que una actitud agresiva sea la forma de presentación de un paciente en el grupo. Es una manera de decir: «cuidado, no me hagas daño». Una paciente se jactaba de haber pegado a un policía. Este tipo de comentarios producen un impacto en los otros y deben ser objeto de atención en el grupo. Se procedió a indagar sobre lo ocurrido, su experiencia, sus sensaciones y a preguntarle sobre la posibilidad de que fuera una defensa ante una situación muy estresante, y la paciente poco a poco fue contando el episodio que vivió en el contexto de una fase maniaca y que refiere que le han contado porque ella no recuerda. Fue muy importante para el grupo aclarar ese comentario y poder entender que la agresividad tenía que ver con la defensa ante el miedo y no con «maldad», ya que esto estaba provocando rechazo en los otros hacia esta persona.

El grupo, obligado por la situación, tiene que hacer el esfuerzo y el aprendizaje de tolerar y contener a otras personas que por su psicopatología interrumpen y no permiten una conversación grupal fluida. Hay ocasiones en que estos pacientes han entrado en el grupo y ya no es momento para ofrecerles salir del mismo, por lo que la situación requiere centrar las intervenciones en este paciente. Los pacientes observan al terapeuta y al personal de apoyo interactuando con esta persona, quienes se convierten en modelos sobre cómo contener. Aroian y Prater (15) señalan que el líder es un modelo de formas de relacionarse de manera empática y efectiva con los pacientes, y así, modela la forma de relacionarse entre ellos. Además, es una oportunidad para que los pacientes puedan ver más allá de los síntomas que tiene una persona. La observación de interacciones respetuosas entre el terapeuta y otros pacientes es una buena base para que cada uno de los pacientes en el grupo se sienta respetado y comience a considerar que su sufrimiento psíquico merece respeto y atención. Muchos pacientes llegan a la Unidad de crisis avergonzados por sus problemas y tratan de suprimirlos cuanto antes sin que les de tiempo a entenderlos. A esto les impulsa además la presión de la unidad para que mejoren.

\section{Permitir la expresión personal de cada paciente}

Durante el desarrollo del grupo, la tarea general del terapeuta es doble: en primer lugar, observar sin ser pasivo la dinámica grupal, los temas relevantes que surgen y merecerán la pena ser tomados como hilo conductor del resto de sesión; y por otra parte, realizar intervenciones adecuadas sobre ese material cuidando de que todos los pacientes tengan un lugar en el proceso grupal.

Para obstaculizar la expresión del paciente, no hay mejor manera que la crítica. En palabras de Yalom (17), este no es lugar para confrontaciones, para la crítica o para la expresión o examen de la ira. 
A veces las defensas caracterológicas de algunos pacientes son muy hirientes para los miembros del grupo. Hay ocasiones en las que el terapeuta tiene que posicionarse ante determinados pacientes. Algunos con rasgos antisociales o marcadamente narcisistas tienen poca probabilidad de beneficiarse del contexto grupal y sin embargo existe un alto riesgo de que «destruyan» la capacidad terapéutica del grupo provocando un intenso sentimiento de inseguridad. Una de las tareas del terapeuta consiste en tener una actitud de protección hacia el grupo y sus miembros.

La disminución de la angustia tiene lugar a medida que los pacientes van conociendo y comprendiendo el lugar en el que están, hablando sobre aspectos tan inusuales en la vida cotidiana como las contenciones, expresando poco a poco toda clase de sentimientos asociados a este espacio y a este momento de sus vidas. Sin embargo, a muchos pacientes les cuesta realizar este proceso por sí mismos. Podemos observarlo con frecuencia en pacientes que reaccionan ante la angustia o incomodidad marchándose del grupo. Cuando un paciente se levanta para marcharse, paramos el grupo para preguntar por qué se quiere ir o si hay alguna cosa que le esté haciendo sentir mal, con el fin de que pueda expresar con palabras su acción de marcharse y facilitar que permanezca dentro del grupo si su angustia no es muy intensa. Hay veces que el paciente que se está yendo no responde y es necesario acercarse a él para llamar su atención y que pueda verbalizar por qué se marcha. El paciente a veces decide quedarse después de verbalizarlo y de que sus opiniones sean tenidas en cuenta (puesto que señalan aspectos del proceso grupal que no estaban apareciendo previamente). Supone por tanto un beneficio para el paciente (aprendizaje de una estrategia de afrontamiento distinta a la evitación) y para el grupo (material emergente).

Cuando un paciente no ha podido expresar su malestar y se marcha del grupo, es muy positivo poder hablar con él unos minutos al terminar la sesión. Ya fuera de la situación ansiógena, el paciente suele expresar al terapeuta las razones de su salida del grupo, con lo que se cierra de alguna manera el proceso y el paciente siente que puede volver al día siguiente.

Es también tarea del terapeuta procurar que todos los pacientes puedan hablar en la sesión. Hay situaciones en que es casi imprescindible que todos los pacientes puedan hablar. Es muy importante que los miembros del grupo puedan tener un espacio para expresar cómo se han sentido ante situaciones en que ha ocurrido o se ha dicho algo muy impactante, con el objetivo no sólo del mero desahogo sino también de la posibilidad de poder trabajarlo dentro del grupo.

\section{Fomentar la parte sana y los recursos personales de cada paciente}

Es tarea del terapeuta reforzar las fortalezas internas y los pequeños cambios que va realizando o viviendo cada paciente. Esto incluye recoger los esfuerzos de 
ORIGINALES Y REVISIONES

mantener el grupo o cooperar como algo muy valioso de la persona. Por ejemplo, ya hemos mencionado la importancia de permitir que sean los propios pacientes los que construyan el grupo y sientan la responsabilidad de hacerlo para su propio interés. Por ejemplo, en una ocasión en la que había diversidad de opiniones en cuanto al tema en el que centrarse en la sesión, un paciente propuso que se hiciera una votación. Esta idea fue recogida por el terapeuta y calificada como una gran aportación al grupo, de tal manera que la tarea de tener que organizarse fue lo significativo de la sesión y no tanto el tema concreto del que podían haber hablado. A partir de la idea de votar, surgió la cuestión de si estaban dispuestos todos a respetar lo que saliera en la votación; posteriormente, si se podían votar varios temas; y una vez terminada la votación y la puesta en práctica de lo votado, algunos pacientes señalan las trasgresiones de lo consensuado («habíamos quedado en no hablar de drogas y al final se ha hablado de drogas»). En este contexto, los pacientes estaban sacando y aprendiendo habilidades de relación, cooperación y negociación, tan necesarias en la vida cotidiana.

Hay ocasiones en que los pacientes no se ven con esa capacidad. A veces es real, pero otras veces no. Esto se observa cuando ante situaciones de mayor desorganización grupal los pacientes culpan al líder por no conseguir que los pacientes hablen en turnos de palabra. La realidad es que el líder no podrá obligar a los pacientes a escucharse unos a otros y a no interrumpirse, por mucho que quiera y proponga actividades estructuradas, si no hay una autorregulación grupal deseada por la mayor parte de los miembros. En estas ocasiones, a menudo basta con señalar esta idea para que el grupo se organice, dando muestras de sus capacidades. Al final del grupo, éstas pueden ser señaladas por el terapeuta.

\section{Dificultades entre pacientes y entre pacientes y personal}

Es natural que entre los pacientes haya conflictos. Se trata de que puedan hablar de ello, para que esos sentimientos asociados al conflicto no sean actuados. El terapeuta debe ayudar a que los conflictos sean expresados con palabras.

Los conflictos relacionales traducen al plano social conflictos intrapsíquicos. Por ejemplo, una paciente comienza la sesión expresando mucho enfado porque otro paciente (presente en el grupo) le había dicho que él era dios e iba a hacer que su padre resucitara (el padre había muerto recientemente, lo que desencadenó el episodio de manía de la paciente), y ella sabía que eso no iba a pasar. A partir de este conflicto entre dos personas, se puede trabajar el duelo de la paciente, quien aun siendo consciente de la cualidad delirante del comentario de su compañero, no podía evitar sentir mucha rabia ante el hecho de que su padre no volvería. La función del terapeuta de grupo aquí es centrar la atención 
en los sentimientos de esta mujer, y no el supuesto conflicto relacional. De hecho, ellos mismos se dan disculpas el uno al otro, se dan la mano, afirman que no quieren pelear. No es tal el conflicto interpersonal, es sólo la señal de que algo le ha tocado internamente a alguien.

En otra sesión, se da un conflicto entre tres pacientes con trastorno bipolar. Una paciente $\left(1 .^{\mathrm{a}}\right)$ «acusa» de forma despectiva a otra paciente $\left(2 .^{\mathrm{a}}\right)$ de estar fingiendo su angustia. La 2 . $^{\mathrm{a}}$ junto con otro paciente $\left(3 .^{\circ}\right)$, también bipolar, se sienten muy heridos y se levantan para marcharse. Se les pide que permanezcan y que expresen con palabras lo que sienten. Aunque están hablando de forma tranquila como se les había pedido, la descalificación continúa, el $3 .^{\circ}$ insulta a la paciente 1. ${ }^{\mathrm{a}}$, quien en ese momento se levanta diciendo que se va porque la insultan. A la $2 .^{a}$, que seguía muy angustiada y que en ningún momento utilizó la descalificación sino una explicación de cómo se encontraba, se le ofrece hablar de su experiencia y al grupo entero se le pregunta por sus habilidades de autoprotección ante comentarios hirientes, que seguramente han sufrido alguna vez fuera de la Unidad. Esta paciente afirma contundente: «Yo no me defiendo», dejándose caer en la silla abatida y llorosa. Realmente, su conducta había sido de «defensa» al invitarla a quedarse y expresarse con la palabra, por lo que se le devuelve esta observación y ella reconoció que efectivamente en esta ocasión había sido así. La paciente pudo ensayar habilidades que creía no tener. Por otra parte, es importante no olvidar al paciente que finalmente se ha marchado e intentar al terminar el grupo hablar con él/ella para valorar su estado y hacer alguna intervención de cierre.

Otro de los asuntos que aparecen en el grupo son desacuerdos sobre las normas, la insatisfacción por la atención (o falta de atención) recibida o las actividades de la unidad. Los desacuerdos sobre las normas son fácilmente abordables si se explicitan claramente y se tiene la oportunidad de aclarar los fundamentos de la existencia de la norma. Los pacientes necesitan tener información clara, aunque no les vaya a gustar, ya sea sobre las normas o sobre cualquier otra cuestión que les vaya a afectar directamente. Por ejemplo: «Sí, hoy es viernes y tu psiquiatra está saliente de una guardia por lo que hoy no te verá, y ya tendrá que ser el lunes». Este tipo de información puede provocar una reacción de enfado que es necesario escuchar, aceptar y contener, siendo estas tres las herramientas más importantes de los conductores del grupo.

Hay que tener en cuenta que los sentimientos de los pacientes de no tener el control, de estar a merced de la estructura a veces rígida de la Unidad, se expresan usualmente en el grupo a través de la rebelión, de luchas por dominar, de hostilidad hacia el terapeuta, el hospital y otros miembros del grupo, por lo que el terapeuta deberá escuchar esos conflictos, señalar que son reacciones normales a la hospitalización y reinterpretarlos como señales de que los pacientes están intentando recuperar el control sobre sus vidas (15). 
ORIGINALES Y REVISIONES

Facilitar el establecimiento y el desarrollo de relaciones nutricias y estructurantes entre los miembros del grupo

El formato grupal posibilita las relaciones interpersonales y constituye una oportunidad de relación para aquellos con tendencia al aislamiento o con falta de iniciativa en el acercamiento a otros. Además, permite enmarcar esa relación en un contexto seguro en el que hay normas explícitas que protegen a sus miembros, lo cual puede facilitar el contacto. Una vez establecido el contacto inicial la experiencia grupal conducirá a los miembros del grupo a experimentar situaciones en las que la matriz de interacciones va a requerir de cada individuo que realice tareas cada vez más complejas y realistas. La tarea del terapeuta es identificar y responder a las necesidades de interacción emergentes que precisan afrontar como resultado de la conexión creciente entre sus miembros. Y ese contacto pretende ser reconfortante, lo cual se promueve ayudando a los pacientes irritantes a obtener apoyo del grupo y evitando la confrontación y el conflicto entre ellos, ya que su estado de vulnerabilidad les dificulta el afrontamiento de los mismos (19).

La forma en que los pacientes se relacionan entre sí dentro del grupo varía mucho dependiendo de factores como su personalidad, su estado psicopatológico, factores extragrupales, y también la fase de desarrollo en la que se encuentre el grupo. Una fase de desarrollo se ha definido en la literatura como un periodo de la vida del grupo en el que los miembros muestran un conjunto específico de conductas sociales hacia los otros que reflejan la preocupación del grupo sobre un tema o conflicto concreto (24). Así, los grupos pasan en su desarrollo por diferentes momentos que requieren que sus miembros realicen diferentes tareas psicológicas, de tal manera que la resolución de las mismas es lo que posibilitará el cambio hacia otra fase. En los grupos abiertos, no siempre se dan todas las fases que habitualmente ocurren en los grupos cerrados, y se observan algunas diferencias dentro de cada fase (1).

Los diferentes modelos propuestos sobre el proceso grupal, aunque difieren en el número de fases, incluyen los siguientes elementos esenciales: una fase de inicio, presentación, toma de contacto y elaboración de objetivos comunes, seguida de una fase de «desencanto»y de cuestionamiento de las figuras de autoridad, tras la cual lograrían crear un sentimiento de pertenencia y confianza en el grupo y sus posibilidades de ayuda más allá del líder, pero contando con su apoyo. Finalmente, se daría una fase de terminación en la que tienen que afrontar la separación.

\section{Ayudar a los pacientes a entender su situación actual}

Es habitual que los pacientes con psicosis digan que no saben por qué han ingresado. Éstos necesitan ser escuchados sin ser confrontados, porque son 
muchas las situaciones en las que reciben el mensaje de que están mal. Sus incógnitas son recogidas y aceptadas como parte del proceso de cada uno, y se plantea el grupo como lugar donde poder encontrar respuesta a esas preguntas. El ingreso es conceptualizado como un paréntesis en sus vidas que llega en un momento determinado y que cumple una función.

Para cubrir este objetivo, pueden ser útiles las técnicas de intervención en crisis, aplicadas a un paciente concreto si se observa que el grupo entero se va a beneficiar o hablando en términos genéricos. Estas técnicas se centran en analizar los pasos que han conducido a la crisis, posibles desencadenantes o factores que han podido influir, recursos psicológicos utilizados, vivencias y emociones particulares durante la crisis, y el abordaje de maneras adaptativas de actuar antes y durante la crisis, así como formas de prevenir la aparición de la crisis en el futuro. Dentro de este proceso, los pacientes suelen manifestar inquietudes tales como el hecho de haber necesitado ingresar en una Unidad de este tipo o sentimientos asociados al alta.

En ocasiones, pacientes con psicosis hablan abiertamente de su enfermedad y de síntomas específicos como delirios y alucinaciones. Es una oportunidad para desmontar mitos o aclarar dudas, también para aquellos que no los padecen o creen no padecerlos. Por ejemplo, en una sesión, un paciente le dice a otro que acaba de decir que tiene esquizofrenia: «No puedes tener eso: estás hablando tan normal». Y más adelante: «iSabes leer y escribir?». Pero sobre todo es una oportunidad para que los pacientes puedan elaborarlo. Generalmente provoca mucha angustia en el grupo escuchar las convicciones delirantes de un paciente. Provoca reacciones muy diversas cuyo origen común es el miedo. Es necesario ayudarles a poder asimilar la experiencia de haber perdido o poder perder el contacto con la realidad. La manera en que se puede hacer es ayudándoles a encontrar un sentido al delirio, transmitir la idea de que el delirio puede ser un recurso para estar en el mundo, de la misma manera en que utilizamos la fantasía o la imaginación para adaptarnos a él.

En todo caso, el terapeuta debe ser sensible a los procesos de duelo que pueden estar poniéndose en marcha, y las diferentes fases del mismo: negación, expresión emocional y aceptación. Virginia Lafond (25), en su artículo sobre el duelo en la enfermedad mental, subraya las malinterpretaciones que se pueden hacer sobre las emociones expresadas por los pacientes en el proceso sano de aceptación de la enfermedad mental, tales como considerarlas como «pérdida de control» o ser incluidas dentro de un diagnóstico y un tratamiento, lo cual puede interferir en la expresión constructiva del duelo. El trabajo del terapeuta, afirma, consistiría en ayudar a las personas a iniciar el proceso de duelo asociado con su enfermedad mental asumiendo una postura afirmativa, es decir, de reconocimiento del esfuerzo y la lucha de la persona por su propia recuperación y una postura docente o de reconocimiento de las emociones asociadas a las pérdidas (tristeza, miedo, rabia) como proceso sano. 
ORIGINALES Y REVISIONES

Por último, no hay mejor forma de que los pacientes puedan entender su situación actual como trabajar el aquí y ahora de la situación grupal. Los pacientes llevan al grupo sus angustias y necesidades, y son elaboradas a propósito de las dinámicas grupales, que como hemos visto están influidas por las fases de desarrollo del grupo. Por ejemplo, cuando los pacientes en una sesión en la que predomina una comunicación caótica, son capaces de hablar de ese caos, comprenderlo y permanecer en el grupo a pesar de todo, están también dándole un sentido y afrontando la desorganización interna.

\section{Abordar cuestiones más profundas}

Dada la continua rotación de pacientes, raramente se dan las condiciones para un trabajo más profundo. Pero a veces ocurre. El grupo que en un momento determinado se forma por los miembros que acuden a las sesiones, está incluido dentro de un sistema que incluye al grupo de todos los pacientes ingresados en la Unidad. Fuera de las sesiones, el proceso grupal continúa, modificándose según nuevos elementos del sistema al cual pertenece, lo cual se verá reflejado a su vez en las siguientes sesiones de grupo. Es un proceso dinámico que se da más allá del grupo de terapia, por lo que muchas veces es posible observar que de hecho existe una cohesión grupal que ha surgido espontáneamente, junto con la influencia del trabajo de los profesionales para favorecer, o no obstaculizar, ese clima terapéutico. En todo caso, se da gracias a que existe una interacción continua fuera de las sesiones.

El abordaje de cuestiones más profundas se ve facilitado si el terapeuta dispone de información previa sobre los conflictos básicos de los pacientes, ya sea de primera mano o a través de coordinaciones con el profesional de referencia que pudieran estar trabajando a esos niveles con el paciente. En una ocasión, el terapeuta del grupo tuvo una sesión individual con un paciente realizada a petición de su psiquiatra con el objetivo de valorar y preparar al paciente para un abordaje psicológico de sus síntomas positivos en el contexto de la atención ambulatoria. El paciente presentaba un delirio erotomaníaco, del cual a veces dudaba. En esa sesión individual, que duró aproximadamente una hora, el paciente conectó de lleno con sentimientos de soledad, llorando verbalizaba lo solo que estaba en la vida, y preguntaba de forma desesperada «¿qué puedo hacer, dime, qué puedo hacer?», a lo que se le respondió: «Ahora estás haciendo mucho; has podido confiar en mí y abrirte y decirme todo esto que estás sintiendo y ahora mismo no estás solo, yo estoy contigo aquí acompañándote». Al final de la entrevista salió reconfortado, aliviado, pero al día siguiente reapareció la irritabilidad, su convicción delirante y su actitud habitual de «no necesito a nadie» y «no pienso ir a un psicólogo, no necesito eso». El paciente había tenido la experiencia de una situación 
de intimidad, que produjo un fuerte miedo inconsciente y volvió a instaurar sus defensas habituales (incluida su convicción delirante).

Unos días después, preguntó en el grupo ¿para qué sirve un psicólogo?, con lo que se produce un debate en el que el paciente niega sistemáticamente que hablar con un psicólogo (u otro profesional) le pueda ayudar. Dado que el terapeuta conocía la capacidad del paciente de poder conectar con sus sentimientos más profundos, y trabajando sobre lo que ocurría en el aquí y ahora, el paciente pudo reconocer que hablar en el grupo le desahogaba y pudo experimentar una sensación de calma al ser escuchado y tenido en cuenta, así como recordar antiguas amistades que había abandonado sin saber por qué.

El trabajo siguiente con este paciente consistiría en ayudarle a asociar sus delirios con sus necesidades básicas, para entender la función de los mismos y darle un sentido coherente con su historia y su forma de sentirse y estar en el mundo, que le pueda ayudar a abandonar viejas defensas en favor de formas de entenderse a sí mismo y de relacionarse más satisfactorias y por tanto a disponer de un nuevo marco de referencia para experimentar esos sentimientos extremadamente angustiantes origen de descompensaciones. Este trabajo suele exceder las posibilidades del grupo de la Unidad, pero quedan asentadas las bases para que otro profesional pueda continuar con esta labor de forma ambulatoria

\section{Superación de obstáculos para el cumplimiento de los objetivos anteriores}

Es importante mencionar que un grupo de estas características plantea muchos retos a los profesionales implicados. Participar en un proceso grupal es una experiencia muy potente que lleva a cuestionarse y replantearse muchos aspectos. Como advierte Yalom (17), conducir un grupo produce ansiedad, y un grupo de psicóticos es en particular intrínsecamente productora de ansiedad. El terapeuta está expuesto a muchas y poderosas, y a menudo, primitivas emociones. El trabajo es lento, a menudo no reconocido y generalmente desconcertante. Los terapeutas están desprotegidos en el sentido de que su trabajo es plenamente visible para un gran número de personas.

Es prioritario que el terapeuta sea consciente de esta ansiedad, y vaya identificando la fuente de la misma y encontrando soluciones, ya que un terapeuta que está extremadamente ansioso no transmitirá la sensación de seguridad y contención que necesitan los pacientes en este contexto. Hay algunas sensaciones típicas en el proceso que el terapeuta y otros profesionales implicados en el grupo tendrán que superar:

- El grupo muchas veces está desorganizado, inquieto, caótico. Como afirma Beeber (16), el terapeuta debe saber que este estado regresivo del grupo 
es predecible y no un defecto. En efecto, es algo que ocurre con frecuencia en los grupos de pacientes ingresados y que el terapeuta debe aceptar, aprender a estar en este caos sin dejarse arrastrar por él y sin entrar en pasividad.

- Es frecuente la sensación de que no tiene sentido el grupo, de que esas sesiones no sirven para nada. Las sesiones son un espejo en el cual se refleja cómo están los pacientes, y no hay que olvidar que están en una situación muy crítica. Los cambios se dan, pero los pacientes necesitan tiempo y una mezcla de ingredientes que dista mucho de poder ser controlada. Pequeños detalles en el grupo pueden suponer una experiencia muy significativa para ellos. La forma de valorar el grupo requiere utilizar criterios basados en la atención a los procesos, y no a los contenidos, para lo cual se necesita entrenamiento.

- Lo que ocurra en la sesión de grupo dependerá de cómo se halle en ese momento preciso y su desarrollo dependerá de las necesidades que sean planteadas consciente o inconscientemente por sus miembros, por lo que hay un alto grado de imprevisibilidad. Es preciso acudir con actitud de observador, sin prejuicios, sin ideas preconcebidas sobre cómo «tiene que» salir el grupo o sobre el material que «tiene que» ser abordado en el mismo. Las expectativas previas son siempre frustrantes y generadoras de ansiedad.

- Por otra parte, se puede caer en el extremo opuesto: no sentirse responsable de nada de lo que ocurra allí y ser pasivo por no saber qué hacer. Si uno no sabe qué hacer, ¡es una cuestión muy interesante para supervisar!

- El terapeuta puede sentir una gran variedad de miedos bien como parte de su historia emocional que se moviliza en el grupo, o como parte de una identificación proyectiva con la experiencia emocional de los miembros. Martínez (14) señala, en relación a situaciones paranoides y violentas entre subgrupos, el miedo a la agresión dentro del grupo, el temor a ser criticado por alguna de las partes en litigio o el miedo a perder el liderazgo.

Hay otras dificultades derivadas de la técnica. El terapeuta tiene que atender a muchos elementos a la vez y extraer de ellos lo relevante y significativo. Supone dirigir la atención hacia los procesos, no sólo de cada individuo dentro del grupo sino también en el nivel grupal. Hay que tener en cuenta que los contenidos pueden ser infinitos (pensemos en la cantidad de cosas que se puede hablar en un grupo) y es fácil perderse en ellos. Por ejemplo, una persona puede estar hablando de la comida y otro de algo que le pasó hace años y estar hablando de lo mismo en términos de «proceso», a un nivel diferente de comunicación (por ejemplo, la necesidad de ser escuchado). Las intervenciones del terapeuta deben conciliar las 
necesidades de la mayoría de los miembros del grupo, y eso requiere una observación muy fina.

Resulta útil valorar lo que dicen los pacientes y aceptar la parte de realidad en que se pueden basar las manifestaciones. Por ejemplo, antes se ha mencionado que una manera de no poner en marcha algunas habilidades relacionales es responsabilizar al líder de lo que está pasando. Es un ejercicio de honestidad valorar si realmente el líder (o la infraestructura hospitalaria) puede hacer algo más o hacer algo diferente para facilitar que esa dinámica se transforme o que efectivamente pongan esas habilidades en marcha.

\section{Conclusión}

La psicoterapia de grupo en las unidades de agudos supone un espacio que posibilita el abordaje de aspectos muy importantes para los pacientes ingresados, tales como las ansiedades derivadas de la hospitalización y las derivadas de su situación de crisis. En los párrafos anteriores se ha presentado una experiencia grupal cuyo objetivo fundamental es ayudar al paciente a recuperar su funcionamiento psicosocial, a través de la psicoterapia de apoyo, sin excluir la posibilidad de abordajes que impliquen profundizar en la psicodinamia de la persona cuando sea posible. Ser contenidos y acogidos, expresarse, seguir poniendo en marcha sus recursos, entender su situación actual y favorecer un clima donde se puedan resolver conflictos interpersonales son algunos de los objetivos específicos del grupo.

Muchos han sido los temas relevantes que los pacientes llevaban al grupo y pudieron recibir allí una respuesta. Los pacientes tienen una necesidad grande de hablar y expresarse, y de ser aceptados y tenidos en cuenta en una situación en la que están tan despojados de su capacidad de autodirección. Comunicar sus estados es una descarga de la angustia imprescindible y la base sobre la cual reconstruirse. Estos son algunos de sus factores terapéuticos, pero hay algunos más; Yalom (27) describió algunos factores terapéuticos de los grupos que sin duda se han encontrado en nuestra experiencia grupal y que hemos ido describiendo: implantar la esperanza; universalidad; impartir información; altruismo; desarrollo de técnicas de socialización; cohesión de grupo.

Y todo esto es posible gracias a la colaboración entre los profesionales de la Unidad, la disposición a emplear la energía en el grupo y a seguir aprendiendo de la experiencia, a través de la continua formación, supervisión y entrenamiento. 
ORIGINALES Y REVISIONES

\section{BIBLIOGRAFÍA}

(1) BRABENDER, V., «Time-limited Inpatient Group Therapy: a Developmental Model», Int. J. Group. Psychother., 1985, 35 (3), pp. 373-390.

(2) Yalom, I. D., Impatient Group Psychotherapy, Nueva York, Basic Books, 1983.

(3) Janssen, P., Psychoanalytic Therapy in the Hospital Setting (Internacional Library of Group Psychotherapy and Group Process), Londres, Routledge, 1994.

(4) Brabender, V.; Fallon, A., Models of Inpatient Group Psychotherapy, Washington D. C., American Psychological Association, 1993.

(5) GAlletero, J. M., y otros, «La psicoterapia de grupo en unidades de hospitalización psiquiátrica de agudos», Avances en Salud Mental Relacional, 2002, 1, 2. Disponible en: http://www.bibliopsiquis.com/asmr/0102/0102lpd.htm (visitado 24/04/08).

(6) Oldham, J. M.; Russakoff, M., Dynamic Therapy in Brief Hospitalization, Northvale, Nueva Jersey, Aronson, 1987.

(7) KiBel, H. D., «Diversity in the Practice of Inpatient Group Psychotherapy in North America», Group Analysis, 1992, 1, 25 (2), pp. 225-239.

(8) Powell, J. E., «Group Psychotherapy for a Heterogeneous Short-Term Inpatient Population», Small Group Research, 1989, 1, 20 (2), pp. 243-258.

(9) FARKAS-CAMERON, M. M., «Inpatient Group Therapy in a Managed Health Care Environment: Application to Clinical Nursing Practice», J. Am. Psychiatr. Nurses Assoc., 1998, 4, 5, pp. $145-152$.

(10) Gauron, E. G., «Inpatient Group Psychotherapy», Am. J. Psychiatry, 1984, 141, pp. 1.2981.299 .

(11) Agazarian, Y. M., A Systems-Centered Approach to Inpatient Group Psychotherapy, Londres, Jessica Kingsley Publishers, 2001.

(12) Clunie, F. S., «In-patient Group Psychotherapy: a Survey of Staff and Patients», Psychiatr. Bull., 1997, 21, pp. 13-15.

(13) Rice, C. A.; RutAN, J. S., Inpatient Group Psychotherapy: A Psychodynamic Perspective, Nueva York, Macmillan, 1987.

(14) MARTínEZ, J. M., «El miedo, las proyecciones paranoides y la violencia en los grupos de pacientes en crisis», Boletín de la Sociedad Española de Psicoterapia y Técnicas de Grupo, 2001, IV, 19, pp. 89-102.

(15) Aroian, K.; Prater, M., «Transition Entry Groups: Easing New Patients' Adjustment to Psychiatric Hospitalization», Hosp. Community Psychiatr., 1988, 39, 3, pp. 312-313.

(16) BeEber, A. R., «A Systems Model of Short-term Open-ended Group Therapy», Hosp. Community Psychiatr., 1988, 39, pp. 537-542.

(17) Yalom, I. D., Psicoterapia existencial y terapia de grupo, Barcelona, Paidós, 2000.

(18) LeSZCZ, M., «Impatient Groups», Ann. Rev. Psychiatry, 1986, 5, p. 729.

(19) Vinogradov, S.; Yalom, I. D., «Terapia de grupo» en Hales, R. E.; YudofsKy, S. C.; TAlbotT, J. A., Tratado de psiquiatría, Washington, American Psychiatric Press, 1988.

(20) KiBEL, H. D., «A Conceptual Model for Short-Term Inpatient Group Psychotherapy», Am. J. Psychiatry, 1981, 138, pp. 74-80.

(21) Martínez, J. M., y otros, «Grupos terapéuticos en unidades de hospitalización de corta estancia», Boletín de la Sociedad Española de Psicoterapia y Técnicas de Grupo, 1997, IV, 11, pp. $139-148$. 
(22) Kaplan, H. I.; SAdock, B. J., Sinopsis de psiquiatría, Madrid, Médica Panamericana, 1999.

(23) BERne, E., Juegos en que participamos. Psicología de las relaciones humanas, México, Diana, 1964.

(24) BRABEnder, V., «A Closed Model of Short-Term Inpatient Group Psychotherapy», Hosp. Community Psychiatr., 1988, 39, pp. 542-545.

(25) LAFOND, V., «La aflicción de la enfermedad mental: contexto para la terapia cognitiva de la esquizofrenia», en Perris, C.; MCGoRRY, P. D. (eds.), Psicoterapia cognitiva para los trastornos psicóticos y de personalidad. Manual teórico práctico, Bilbao, DDB, 2004.

(26) BERNE, E., Introducción al tratamiento de grupo, Barcelona, Grijalbo, 1983.

(27) Yalom, I. D., Teoría y práctica de la psicoterapia de grupo, México, FCE, 1986.

* Beatriz Martín Cabrero, psicóloga Clínica. Unidad de Psiquiatría del Hospital Son Llàtzer (Palma de Mallorca); José Manuel Martínez Rodríguez, psiquiatra. Jefe de Servicio de Asistencia Psiquiátrica. Gerencia Regional de Salud de Castilla y León.

Correspondencia: Beatriz Martín Cabrero. Ctra Manacor km. 4. 07198 Palma de Mallorca. Tlf. 629714084.

** Fecha de recepción: 9-VII-2008 (aceptado el 10-VIII-2008). 